\title{
Protocol of a systematic review on telemedicine solutions in COVID-19 pandemic
}

\author{
Saeid Eslami1 ${ }^{1,2,3}$ (iD), Raheleh Ganjali1,4*(D) \\ ${ }^{1}$ Department of Medical Informatics, Faculty of Medicine, Mashhad University of Medical Sciences, Mashhad, Iran \\ ${ }^{2}$ Pharmaceutical Research Center, Mashhad University of Medical Sciences, Mashhad, Iran \\ ${ }^{3}$ Department of Medical Informatics, University of Amsterdam, Amsterdam, the Netherlands \\ ${ }^{4}$ Clinical Research Development Unite, Emam Reza Hospital, Mashhad University of Medical Sciences, Mashhad, Iran
}

\begin{tabular}{ll}
\hline Article Info & A B S T R A C T \\
\hline $\begin{array}{l}\text { Article type: } \\
\text { Review }\end{array}$ & Introduction: On March 20, 2020, the World Health Organization (WHO) \\
& announced the spread of SARS-CoV-2 infection in most countries worldwide \\
& as a pandemic. COVID-19 is mainly disseminated through human-to-human \\
transmission route via direct contact and respiratory droplets. Telehealth & and/or telemedicine technologies are beneficial methods that could be \\
Article History: & employed to deal with pandemic situation of communicable infections. The \\
Received: $2021-06-27$ & purpose of this proposed systematic review study is to sum up the \\
Accepted: $2021-10-19$ & functionalities, applications, and technologies of telemedicine during \\
Published: $2021-11-01$ & COVID-19 outbreak.
\end{tabular}

\section{Raheleh Ganjali}

Clinical Research Development Unite, Emam Reza Hospital, Mashhad University of Medical Sciences, Mashhad, Iran

Email: Ganjalir2@mums.ac.ir

\section{Keywords:}

Tele-Medicine Services (TMS) COVID-19

Systematic Review

Quality Assessment
Material and Methods: This review will be carried out in accordance with the Cochrane Handbook and PRISMA (Preferred Reporting Items for Systematic Reviews and Meta-Analyses) reporting guidelines. PubMed and Scopus databases were searched for related articles. Randomized and nonrandomized controlled trials published in English in scientific journals were identified to be evaluated for eligibility. Articles conducted on telemedicine services (TMS) during COVID-19 outbreak (2019-2020) were identified to be evaluated.

Results: The literature search for related articles in PubMed and Scopus databases led to the identification and retrieval of a total of 1118 and 485 articles, respectively. After eliminating duplicate articles, title and abstract screening process was performed for the remaining 1440 articles. The current study findings are anticipated to be used as a guide by researchers, decision makers, and managers to design, implement, and assess TMS during COVID-19 crisis.

Conclusion: As far as we know, this systematic review is conducted to comprehensively evaluate TM methods and technologies developed with the aim of controlling and managing COVID-19 pandemic. This study highlights important applications of telemedicine in pandemic conditions, which could be employed by future health systems in controlling and managing communicable infections when an outbreak occurs.

Cite this paper as:

Eslami S, Ganjali R. Protocol of a systematic review on telemedicine solutions in COVID-19 pandemic. Front Health Inform. 2021; 10: 96. DOI: $10.30699 /$ fhi.v10i1.317

\section{INTRODUCTION}

The number of COVID-19 cases in the world has increased rapidly, [1]]. On March 20, 2020, the World Health Organization (WHO) announced the spread of SARS-CoV-2 infection in most countries worldwide as a pandemic [2] . COVID-19 is mainly disseminated through human-to-human transmission route via direct contact and respiratory droplets [ [ $]$ ]. This large-scale pandemic disease poses many problems for health systems around the world, harms endangered populations, and threatens the global health of human communities in an unexampled manner [4]. The reports have been showed more than five million people affected to COVID-19 in Iran until September 2021. Due to the lack of an effective therapeutic treatment for SARS-CoV-2 infection, healthcare systems primarily focus on symptomatic therapy, social distance, the use of masks for personal 
protection, and forced quarantine after exposure to an infected person [ [ $]$. However, hospitalized patients not infected with COVID-19, especially those at higher risk of developing the disease (such as the elderly and those with underlying diseases), should receive daily care with minimal contact with other patients [6]. On the other hand, unnecessary medical staff such as clinical psychiatrists should be strictly prevented from entering COVID-19 ward where patients are under strict infection control $[\underline{7}, \underline{8}]$. Natural catastrophes and epidemics create many problems for healthcare systems in providing health services [9]. However, the new pandemic has led to the widespread application of telehealth for facilitating patient-healthcare provider interaction $[\underline{5}, \underline{10}]$, so that medical visits have shifted from faceto-face to remote medical appointments. Thus, telehealth and/or telemedicine technologies are beneficial methods that could be employed to deal with pandemic situation of communicable diseases. The benefits of using such technologies to manage calamities and pandemics have been well documented $[\underline{11}, \underline{12}]$.

The term telemedicine, invented in the 1970s, conveys the meaning of "healing at a distance" [13]. Over the past 4 decades, many peer-reviewed definitions have been suggested for this term. The standardized definition proposed by the WHO in 2007 for the term telemedicine is the provision of remote healthcare services by healthcare providers through employing information and communication technologies (ICT) to detect, treat, and prevent diseases and injuries, to investigate and assess the topic of interest, and to teach health personnel with the aim of improving people health [14]. ICTs are technological instruments and resources employed for transferring, collecting, creating, and exchanging data, including computers, internet (websites, blogs, and emails), live (radio, television, and webcasting) and recorded (podcasting, audio and video players, and storage devices) broadcasting technologies, and telephony (fixed or mobile, satellite, videoconferencing, etc.) [15]. The terms telemedicine and telehealth are occasionally used correspondingly by researchers [16]. However, there is a little distinction between the two. Telehealth is a more general term compared to telemedicine, denoting both remote clinical and non-clinical (such as administrative meetings) services, while the term telemedicine is usually used to refer to only remote clinical services [16].

The primary advantage of using telemedicine is to decrease or remove geographical distances [17]. The development of high-speed telecommunication networks and transfer of high-quality images and other clinical data have further empowered telemedicine while reducing costs and travel time for patients $[\underline{18}, \underline{19}]$.
Several review studies have explained the use of telemedicine and its dimensions in COVID-19 outbreak $[\underline{1}, \underline{5}, \underline{20}-\underline{22}]$. Thus, this systematic review study aims to determine and sum up the functionalities, applications, and technologies of telemedicine in COVID-19 crisis. The results of which could be used as a guide by researchers to design and implement telemedicine in catastrophic situations.

In the following section, research questions are raised, and some items are defined, including the study population, the type of intervention used, the control used for comparing the findings, and the outcome framework used for identifying and combining Medical Subject Headings (Mesh), subject headings, and keywords.

\section{MATERIAL AND METHODS}

The Cochrane protocol guideline was pursued to develop this systematic review protocol [23]. Also, the Preferred Reporting Items for Systematic Reviews and Meta Analyses (PRISMA) will be followed during this study procedures to further ensure research rigor. In addition, PRISMA protocol (PRISMA-P) 2015 checklist [24, 25] was employed to report this systematic review protocol.

The research team is composed of healthcare and medical informatics (MI) specialists who are experienced in research and education and have clinical experience in MI.

\section{Research questions}

This systematic review study aims to determine and sum up the functionalities, applications, and technologies of TMS during COVID-19 pandemic. The effect of TM would be explained in terms of one or more of three metrics. The following research questions are addressed in this study:

RQ1: Which studies have employed TM services in COVID-19 pandemic?

RQ2: What are the functionalities of TM in these studies?

RQ3: What are the most frequently used TM applications in these studies?

The participants in all studies included are human subjects. The intervention is telemedicine application, and the control is conventional healthcare techniques. An outcome is considered as positive if telemedicine helps the health system in detecting, treating (including follow up), and/or managing the pandemic.

\section{Searches}

The following sources were searched for primary studies: 


\section{- $\quad$ MEDLINE, via PubMed}

- Scopus

The search terms were detected based on two main concepts, including telemedicine and its associated concepts (behavior or phenomenon of interest) as well as COVID-19 (health context). A combination of keywords and Mesh terms (for PubMed and Scopus) associated with telemedicine and COVID-19, which were determined in the preliminary searches, were used to identify related articles. There was no limitation on the publication date of articles, but based on the inclusion criteria, only those articles published in English were selected. Search strategies were developed, and search terms were selected according to each database. The literature search results were downloaded and imported into EndNote X8.2 (Thomson Reuters, NY). Endnote was used to identify articles meeting the pre-specified inclusion criteria. Duplicate articles were detected and eliminated using the Endnote duplicate tool. Duplicates not recognized by the Endnote were manually identified and deleted.

\section{Eligibility criteria}

Studies will be selected according to the criteria related to the following areas, including study population, type of intervention, comparator, desired outcome(s), and study design (PICOS framework)[드. These areas are described in detail as follows:

- Participants: Studies involving patients receiving TMS will be selected, including ambulatory patients (those patients needing to medical services but not as in person follow-up, visit, or consultation), emergency patients (those patients needing to necessary clinical services), or in-patients (those patients involved in disease diagnose or treatment in hospital).

- Interventions: Telemedicine is described by the WHO as the delivery of remote health services by employing electronic tools to detect, treat, and prevent diseases and injuries, to investigate and assess the topic of interest, and to teach health personnel [27]. Telemedicine is currently considered as one of the most effective methods in the development of modern health systems for the detection/diagnosis, therapy, and prevention of diseases [28]. Conceptually, the association of telemedicine to telehealth is the same as the association of medicine to health. Medical care and public health are the closest practical domains that well illustrate the association between these two concepts, but others may lead to a different conceptualization. In any case, the term telehealth to some extent reflects the correctness of a country's public affairs in modern discourse, which is also evident in a more comprehensive term in medicine [29]. Telemedicine technology could range from telephone consultation (in comparison with face-to-face consultation as a common treatment) to videoconferencing consultation (in comparison with telephone consultation).

- Desired outcome(s): Studies obtaining at least one outcome will be included in this review. The primary outcomes are those outcomes associated to patients, and the secondary outcomes are health outcomes reported in articles by investigators.

- Study design: Eligible studies will be randomized and non-randomized controlled trials in which TM is the primary intervention used. Non-randomized studies consist of case-control, cohort, and crosssectional studies, while randomized studies consist of randomized controlled trials and quasi experimental studies.

\section{Exclusion criteria}

Reviews, editorials/commentaries, and methodological articles will be excluded. No restrictions will be imposed on the publication status, and articles, reports, and unpublished studies will be considered as eligible. Studies published in English in scientific journals during 2019 to 2020 will be taken into account. Studies conducted on the design, applicability, or description of IT-based tools without assessing any outcomes, studies conducted on the efficacy of a single mobile device, and electronic health records will be excluded.

\section{Selection process}

Selection of eligible articles will be done manually in three steps by taking into account the inclusion/exclusion criteria as follows:

1. Articles titles will be screened by one investigator, and the results will be checked by another investigator for consistency.

2. Articles abstracts will be reviewed by two investigators, and the results will be checked for consistency.

3. Articles full-text evaluation will be performed by two investigators, and the results will be checked for consistency. In cases where there is disagreement between investigators, agreement will be sought by discussion or by including a third investigator to reach a consensus.

A PRISMA flowchart will be employed to illustrate the 
process of identifying and screening articles to be included in this systematic review as well as to show the reasons for excluding other items [을.

\section{Data extraction}

Data extraction will be performed by one reviewer and checked by a second reviewer. Any disagreement will be resolved by reaching a consensus via discussion or including a third reviewer. Data will be extracted using a standardized form. The following data with be extracted from the articles, including title, year and month of publication, aims and objectives, country, participant characteristics, intervention, and outcome measure(s).

\section{Reporting quality in individual studies}

To evaluate the risk of bias, the guideline of observational studies (cohort, cross-sectional, and case-control) will be followed by employing the STROBE tool [31]. Quality assessment will be performed for quasi-experimental studies by employing the Quality Assessment Tool, developed by Brown for pre- and post-intervention study design according to another study by Estabrooks et al. [프, 33].

\section{Data synthesis}

A brief description of the PICO elements and included studies results will be provided in two different tables exhibiting the summary of bibliographic data, methods used, outcomes, and conclusions at the study level. Articles will also be classified and enumerated in a two-way matrix based on the context and the type of technological intervention applied. Then outcome measures will be collated as second-tier descriptions and classified into clinical efficacy measures or service utilization measures.

\section{RESULTS}

In this review study, all papers selected were published from 2019 to 2020 at the same time as the outbreak of COVID-19. The literature search for the related articles in PubMed and Scopus databases led to the identification and retrieval of a total of 1118 and 485 articles, respectively (Fig 1). Then databases search results were imported into Endnote. After eliminating 163 duplicate articles, title and abstract screening process was performed for the remaining 1440 articles.

The results of this systematic review provide a comprehensive outlook of telemedicine functionalities and technologies designed with the aim of controlling COVID-19 outbreak. The present study results are anticipated to be used as a guide by researchers, decision makers, and managers to design, implement, and assess telemedicine services in order to help medical personnel and patients in fighting and eliminating COVID-19.

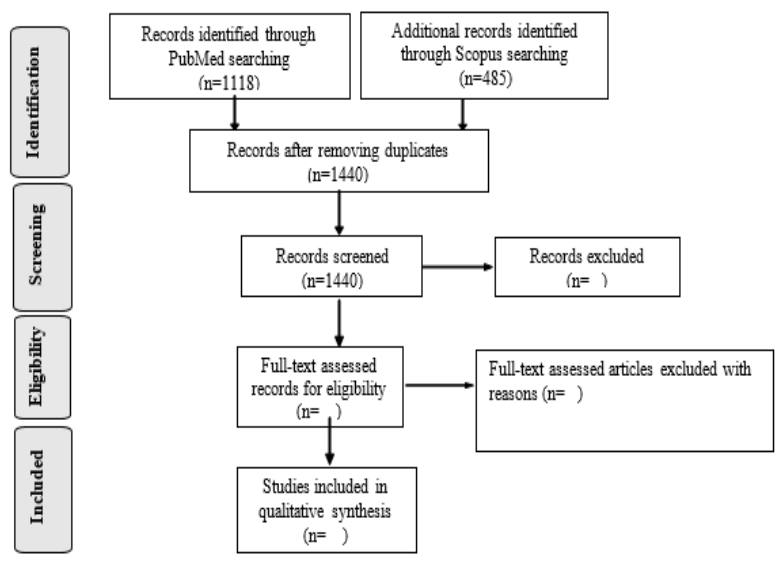

Fig 1: Flow diagram of the literature search and publications selection

\section{DISCUSSION}

This systematic review will be conducted with the aim of determining and summarizing the functionalities, applications, and technologies of telemedicine in COVID-19 outbreak. Furthermore, this study aims to provide a comprehensive outlook of telemedicine domains, functionalities, applications, and technologies as well as clinical areas participating in this field. This systematic review will be performed in accordance with PRISMA guidelines. In fact, the major purpose of this review is to determine which technology in which clinical context could be employed to assist in managing and controlling COVID-19 pandemic. Using a wide range of keywords and Mesh terms to develop the search strategy could be considered as a strength point of this review. However, the use of a framework to classify and describe telemedicine applications and technologies is considered as the strength point of this study. On the other hand, insights and judgements of reviewers may affect the results; however, multidisciplinary viewpoints and the extensive experience of reviewers are deemed to enhance the worthiness of the synthesized data in this study. Systematic review studies are believed to enhance knowledge and scientific techniques required to develop and evaluate TM technologies and instruments.

\section{CONCLUSION}

As far as we know, this systematic review is the first study conducted to comprehensively evaluate TM methods and technologies designed with the aim of controlling and managing COVID-19 outbreak. This study highlights important applications of telemedicine in pandemic conditions, which could be employed by future health systems in controlling and managing communicable infections when an outbreak occurs. 


\section{AUTHOR'S CONTRIBUTION}

RG conceptualized and coordinated the study and drafted the manuscript. SE contributed to the design and reviewed the contributions.

All authors contributed to the literature review, design, data collection and analysis, drafting the manuscript, read and approved the final manuscript.

\section{REFERENCES}

1. Hikmahwati WS. A systematic review: Challenges and evaluations related to telemedicine as a healthcare's hope to tackle COVID-19. International Conference of Health Development Covid-19 and the Role of Healthcare Workers in the Industrial Era. Atlantis Press; 2020.

2. Matamala-Gomez M, Bottiroli S, Realdon O, Riva G, Galvagni L, Platz T, et al. Telemedicine and virtual reality at time of COVID-19 pandemic: An overview for future perspectives in neurorehabilitation. Front Neurol. 2021; 12: 646902. PMID: 33841313 DOI: 10.3389/fneur.2021.646902 [PubMed]

3. Zheng S-Q, Yang L, Zhou P-X, Li H-B, Liu F, Zhao R-S. Recommendations and guidance for providing pharmaceutical care services during COVID-19 pandemic: a China perspective. Res Social Adm Pharm. 2021; 17(1): 1819-24. PMID: 32249102 DOI: 10.1016/j.sapharm.2020.03.012 [PubMed]

4. Ghosh A, Gupta R, Misra A. Telemedicine for diabetes care in India during COVID19 pandemic and national lockdown period: Guidelines for physicians. Diabetes Metab Syndr. 2020; 14(4): 273-6. PMID: 32283497 DOI: 10.1016/j.dsx.2020.04.001 [PubMed]

5. Bitar H, Alismail S. The role of eHealth, telehealth, and telemedicine for chronic disease patients during COVID-19 pandemic: A rapid systematic review. Digit Health. 2021; 7: 20552076211009396. PMID: 33959378 DOI: $10.1177 / 20552076211009396$ [PubMed]

6. Smith AC, Thomas E, Snoswell CL, Haydon H, Mehrotra A, Clemensen J, et al. Telehealth for global emergencies: Implications for coronavirus disease 2019 (COVID-19). J Telemed Telecare. 2020; 26(5): 309-13. PMID: 32196391 DOI: 10.1177/1357633X20916567 [PubMed]

7. Kang L, Li Y, Hu S, Chen M, Yang C, Yang BX, et al. The mental health of medical workers in Wuhan, China dealing with the 2019 novel coronavirus. Lancet Psychiatry. 2020; 7(3): e14. PMID: 32035030 DOI: 10.1016/S2215-0366(20)30047-X [PubMed]

8. Li W, Yang Y, Liu Z-H, Zhao Y-J, Zhang Q, Zhang L, et al. Progression of mental health services during the COVID-19 outbreak in China. Int J Biol Sci. 2020; 16(10): 1732-8. PMID: 32226291 DOI: 10.7150/ijbs.45120 [ubMed]

9. Chauhan V, Galwankar S, Arquilla B, Garg M, Di Somma S, El-Menyar A, et al. Novel coronavirus (COVID-19):

\section{CONFLICTS OF INTEREST}

The authors declare no conflicts of interest regarding the publication of this study.

\section{FINANCIAL DISCLOSURE}

This study was supported by a grant from Mashhad University of Medical Sciences (990447) Research Council.

Leveraging telemedicine to optimize care while minimizing exposures and viral transmission. J Emerg Trauma Shock. 2020; 13(1): 20-4. PMID: 32308272 DOI: 10.4103/JETS.JETS_32_20 [PubMed]

10. Wittenberg E, Goldsmith JV, Chen C, Prince-Paul M, Johnson RR. Opportunities to improve COVID-19 provider communication resources: A systematic review. Patient Educ Couns. 2021; 104(3): 438-51. PMID: 33455825 DOI: 10.1016/j.pec.2020.12.031 [PubMed]

11. Hollander JE, Carr BG. Virtually perfect? Telemedicine for COVID-19. N Engl J Med. 2020; 382(18): 1679-81. PMID: 32160451 DOI: 10.1056/NEJMp2003539 [PubMed]

12. Lurie N, Carr BG. The role of telehealth in the medical response to disasters. JAMA Intern Med. 2018; 178(6): 745-6. PMID: 29710200 DOI: 10.1001/jamainternmed.2018.1314 [PubMed]

13. Strehle E, Shabde N. One hundred years of telemedicine: Does this new technology have a place in paediatrics? Arch Dis Child. 2006; 91(12): 956-9. PMID: 17119071 DOI: 10.1136/adc.2006.099622 [PubMed]

14. Kruse CS, Krowski N, Rodriguez B, Tran L, Vela J, Brooks M. Telehealth and patient satisfaction: A systematic review and narrative analysis. BMJ Open. 2017; 7(8): e016242. PMID: 28775188 DOI: 10.1136/bmjopen-2017-016242 [PubMed]

15. UNESCO. Guide to measuring information and communication technologies (ICT) in education [Internet]. 2009 [cited: 1 Agu 2021]. Available from: http://uis.unesco.org/sites/default/files/documents /guide-to-measuring-information-andcommunication-technologies-ict-in-educationen_0.pdf

16. Fatehi F, Wootton R. Telemedicine, telehealth or ehealth? A bibliometric analysis of the trends in the use of these terms. J Telemed Telecare. 2012; 18(8): 4604. PMID: 23209265 DOI: 10.1258/jtt.2012.gth108 [PubMed]

17. Neville CW. Telehealth: A balanced look at incorporating this technology into practice. SAGE Open Nurs. 2018; 4: 2377960818786504. PMID: 33415198 DOI: $10.1177 / 2377960818786504$ [PubMed]

18. Ellimoottil C, Skolarus T, Gettman M, Boxer R, Kutikov A, Lee BR, et al. Telemedicine in urology: State of the art. Urology. 2016; 94: 10-6. PMID: 27109596 DOI: 10.1016/j.urology.2016.02.061 [PubMed] 
19. Hersh W, Helfand M, Wallace J, Kraemer D, Patterson P, Shapiro S, et al. A systematic review of the efficacy of telemedicine for making diagnostic and management decisions. J Telemed Telecare. 2002; 8(4): 197-209. PMID: 12217102 DOI: 10.1258/135763302320272167 [PubMed]

20. Andrews E, Berghofer K, Long J, Prescott A, CaboralStevens M. Satisfaction with the use of telehealth during COVID-19: An integrative review. Int J Nurs Stud Adv. 2020; 2: 100008. PMID: 33083791 DOI: 10.1016/j.ijnsa.2020.100008 [PubMed]

21. de Oliveira Andrade A, Soares AB, de Andrade Palis A, Cabral AM, Barreto CGL, de Souza DB, et al. On the use of telemedicine in the context of COVID-19: Legal aspects and a systematic review of technology. Clin Kidney J. 2020; 14(2): 492-506. PMID: 33619442 DOI: $10.1093 / \mathrm{ckj} / \mathrm{sfaa} 103$ [PubMed]

22. Kichloo A, Albosta M, Dettloff K, Wani F, El-Amir Z, Singh J, et al. Telemedicine, the current COVID-19 pandemic and the future: A narrative review and perspectives moving forward in the USA. Fam Med Community Health. 2020; 8(3): e000530. PMID: 32816942 DOI: $10.1136 /$ fmch-2020-000530 [PubMed]

23. Higgins JP, Green S. Cochrane handbook for systematic reviews of interventions version 5.1.0 [Internet]. 2011 [Cited: 15 Aug 2021]. Available from: https://handbook-5-1.cochrane.org/

24. Moher D, Shamseer L, Clarke M, Ghersi D, Liberati A, Petticrew $M$, et al. Preferred reporting items for systematic review and meta-analysis protocols (PRISMA-P) 2015 statement. Syst Rev. 2015; 4(1): 1. PMID: 25554246 DOI: 10.1186/2046-4053-4-1 [PubMed]

25. Shamseer L, Moher D, Clarke M, Ghersi D, Liberati A, Petticrew $M$, et al. Preferred reporting items for systematic review and meta-analysis protocols (PRISMA-P) 2015: Elaboration and explanation. BMJ. 2015; 350: g7647. PMID: 25555855 DOI: 10.1136/bmj.g7647 [PubMed]

26. Stern C, Jordan Z, McArthur A. Developing the review question and inclusion criteria. Am J Nurs. 2014; 114(4): 53-6. PMID: 24681476 DOI: 10.1097/01.NAJ.0000445689.67800.86 [PubMed]

27. Serper M, Volk ML. Current and future applications of telemedicine to optimize the delivery of care in chronic liver disease. Clin Gastroenterol Hepatol. 2018; 16(2): 157-61. PMID: 29389489 DOI: 10.1016/j.cgh.2017.10.004 [PubMed]

28. Alonso SG, Marques G, Barrachina I, Garcia-Zapirain B, Arambarri J, Salvador JC, et al. Telemedicine and eHealth research solutions in literature for combatting COVID-19: A systematic review. Health Technol (Berl). 2021; 1-10. PMID: 33558838 DOI: 10.1007/s12553-021-00529-7 [PubMed]

29. Bashshur R, Shannon G, Krupinski E, Grigsby J. The taxonomy of telemedicine. Telemed J E Health. 2011; 17(6): 484-94. PMID: 21718114 DOI: 10.1089/tmj.2011.0103 [PubMed]

30. Moher D, Liberati A, Tetzlaff J, Altman DG. Preferred reporting items for systematic reviews and metaanalyses: The PRISMA statement. BMJ. 2009; 339: b2535. PMID: 19622551 DOI: 10.1136/bmj.b2535 [PubMed]

31. Vandenbroucke JP, von Elm E, Altman DG, Gøtzsche PC, Mulrow CD, Pocock SJ, et al. Strengthening the reporting of observational studies in epidemiology (STROBE): Explanation and elaboration. PLoS Med. 2007; 4(10): e297. PMID: 17941715 DOI: 10.1371/journal.pmed.0040297 [ $\underline{\text { PubMed] }}$

32. Estabrooks C, Goel V, Thiel E, Pinfold P, Sawka C, Williams I. Decision aids: Are they worth it? A systematic review. J Health Serv Res Policy. 2001; 6(3): 170-82. PMID: 11467275 DOI: $10.1258 / 1355819011927431$ [PubMed]

33. Estabrooks CA, Cummings GG, Olivo SA, Squires JE, Giblin C, Simpson N. Effects of shift length on quality of patient care and health provider outcomes: Systematic review. Qual Saf Health Care. 2009; 18(3): 181-8. PMID: 19467999 DOI: 10.1136/qshc.2007.024232 [PubMed] 